Original Articles

\title{
Vision dominates in perceptual language: English sensory vocabulary is optimized for usage
}

\author{
Bodo Winter $^{\mathrm{a}, *}$, Marcus Perlman ${ }^{\mathrm{a}}$, Asifa Majid ${ }^{\mathrm{b}, \mathrm{c}, \mathrm{d}}$ \\ ${ }^{a}$ University of Birmingham, Dept. of English Language and Applied Linguistics, United Kingdom \\ b Centre for Language Studies, Radboud University, Nijmegen, The Netherlands \\ ${ }^{c}$ Max Planck Institute for Psycholinguistics, Nijmegen, The Netherlands \\ ${ }^{\mathrm{d}}$ Donders Institute for Brain, Cognition and Behaviour, Nijmegen, The Netherlands
}

\section{A R T I C L E I N F O}

\section{Keywords:}

Sensory words

Perception

Sight

Lexicon

Word frequency

Embodied cognition

\begin{abstract}
A B S T R A C T
Researchers have suggested that the vocabularies of languages are oriented towards the communicative needs of language users. Here, we provide evidence demonstrating that the higher frequency of visual words in a large variety of English corpora is reflected in greater lexical differentiation-a greater number of unique words-for the visual domain in the English lexicon. In comparison, sensory modalities that are less frequently talked about, particularly taste and smell, show less lexical differentiation. In addition, we show that even though sensory language can be expected to change across historical time and between contexts of use (e.g., spoken language versus fiction), the pattern of visual dominance is a stable property of the English language. Thus, we show that across the board, precisely those semantic domains that are more frequently talked about are also more lexically differentiated, for perceptual experiences. This correlation between type and token frequencies suggests that the sensory lexicon of English is geared towards communicative efficiency.
\end{abstract}

\section{Introduction}

The vocabularies of languages appear to be geared towards the communicative needs of their speakers. In the domain of color, for example, Berlin and Kay (1969) famously suggested that languages only have a small set of color words that tend to cluster around similar perceptual foci across languages (see also Cook, Kay, \& Regier, 2005). Indeed, recent evidence indicates that basic color terms are not randomly distributed across the color spectrum, but rather partition it in a way that is most efficient to refer to colors in human environments. Griffin (2006) showed computationally that basic color terms such as red, blue and green produce better color categorization performance of natural images than other color categorization systems, while Yendrikhovskij (2001) found that natural image statistics reveal color clusters closely aligned with the color terms frequently found in the world's languages. Similarly, Gibson et al. (2017) showed speakers more commonly talk about warm-colored objects in the world, and consequently languages have more dedicated means to talk about 'warm' colors.

Similar adaptations to language use have been demonstrated in other conceptual domains. One linguistic "signature" of being geared towards efficiency in usage is when so-called "type" and "token" frequencies are correlated with each other (e.g., Regier, Carstensen, \& Kemp, 2016). Type frequencies measure the number of unique word types within a given domain, i.e., how lexically differentiated a domain is. Token frequencies measure how frequently each unique word type is used. A positive correlation between type and token frequencies across conceptual domains indicates that the lexicon of a language has more words precisely for those concepts that speakers also talk about more frequently. Moreover, a correlation between type and token frequency is doubly impressive because if a conceptual domain is broken up into more distinct word types, we may expect each type to be less frequent. If, however, type and token frequencies are positively correlated, then this indicates an even greater need to talk about a given conceptual domain. Regier et al. (2016) showed that languages spoken in relatively colder climates are more likely to distinguish between the concepts 'ice' and 'snow' (type frequencies), and they also more frequently refer to these concepts (token frequencies of both types). On the other hand, languages spoken in warmer climates are more likely to collapse the ice/snow distinction. Similarly, Warriner and Kuperman (2015) showed that English speakers use positive words such as pleasure more frequently than negative words such as disgust, and they similarly showed that the English language also has more distinct positive word types in the lexicon.

\footnotetext{
* Corresponding author.

E-mail address: bodo@bodowinter.com (B. Winter)
} 
These studies demonstrate how the lexicons of English and other languages are geared towards communicating effectively about various conceptual domains, such as color and ice/snow. Here, we investigate how English is optimized for communicating about our sensory experience more generally. In particular, evidence from different disciplines, including cognitive psychology (reviewed in Stokes \& Biggs, 2015), anthropology (e.g., Classen, 1993, 1997), linguistics (e.g., Levinson \& Majid, 2014; Viberg, 1983), and philosophy (Keller, 2016; Korsmeyer, 1999), suggests that vision is the most important sensory modality, at least in Western cultures (Majid et al., in press). Our study examines whether the structure of the English lexicon and the way it is used corresponds to this visual dominance in perception. Does English feature more words for visual concepts compared to the other senses? And do speakers use these words more frequently?

\subsection{Visual dominance in perception}

The hypothesis that English is optimized for the communication of visual concepts is based on multiple strands of evidence which together suggest that vision is the dominant human sense. In particular, demonstrations of "visual dominance" in perception (for review see Stokes \& Biggs, 2015) are persuasive. For example, in the so-called "ventriloquist effect", the location where something is seen overrides the location where something is heard (Alais \& Burr, 2004; Pick, Warren, \& Hay, 1969; Welch \& Warren, 1980). Additionally, the influence of vision extends to the other senses: How something is seen modulates how something is felt more strongly than the other way around (Hay \& Pick, 1966; Rock \& Victor, 1964), and vision can also influence how something is tasted or smelled (Hidaka \& Shimoda, 2014; Morrot, Brochet, \& Dubourdieu, 2001; Shermer \& Levitan, 2014). These studies demonstrate the capacity of vision to profoundly affect how the other sensory modalities are perceived, more so than the reverse. Moreover, when people integrate information across senses, visual information is often privileged over other sensory modalities (e.g., Spence, Parise, \& Chen, 2012). People also find it easier to perform mental imagery in the visual modality than in other modalities (e.g., Brower, 1947; Kosslyn, Seger, Pani, \& Hillger, 1990). Finally, visual dominance is arguably indicated in the anatomy of the human brain, with studies suggesting that vision occupies the largest part of cortex (Drury et al., 1996; Palmer, 1999).

\subsection{Visual dominance reflected in language}

Given the dominance of vision in perception, the hypothesis that languages adapt to communicative need predicts that languages should be geared towards talking about visual concepts, compared to the other senses. Indeed, in linguistics, the idea that language may exhibit visual dominance is not a new one (see Levinson \& Majid, 2014), with Buck (1949: chap. 15) already noting in his Indo-European dictionary that for English verbs, there are more agency distinctions for the visual (to see, to look, to look at) and auditory modality (to hear, to sound, to listen) than for the gustatory and olfactory modalities. For example, an English speaker lexically distinguishes between it looked good and she saw it, but not between it smelled good and she smelled it. This work was extended by Viberg (1983), who demonstrated that across several languages and language families, verbs of visual perception are indeed more lexically differentiated than perceptual verbs for other sensory modalities (see also Evans \& Wilkins, 2000). Other researchers have argued that visual verbs are also more likely to be semantically extended compared to verbs for the other sensory modalities, as when speakers say I see you to mean 'I understand you' (see Caplan, 1973; Matlock, 1989; Evans \& Wilkins, 2000; Sweetser, 1990; Ibarretxe-Antuñano, 2008). In addition, Viberg (1993) showed that visual verbs in English have higher token frequencies in text corpora, a finding that was extended to everyday conversation across 13 different languages by San Roque et al. (2015).

\subsection{Current study}

These studies are consistent with the hypothesis that visual dominance in perception and human behavior corresponds to visual dominance in the vocabularies of English and other languages, such that there are a greater array of verbs for vision-related concepts. Moreover, speakers use these different verbs more frequently than those of the other, less differentiated senses. But just how deep and pervasive is the perceptual dominance of vision in language?

In the current study, we tested whether visual dominance in English extends across the sensory vocabulary, including adjectives such as blue, soft and fragrant, and nouns such as music and reflection. Our analysis also spans multisensory words such as large and harsh, which clearly describe perceptual content, but not perceptual content exclusive to just one modality (see Lynott \& Connell, 2009; Winter, 2016b: chap. 2). We also examined whether visual dominance was robust across registers-such as fiction or academic writing-and whether the pattern is stable across time. Across our analyses, we investigated both unique types of words, as well as their token frequencies. Our results show-across lexical class, register, and historical time-that the English language contains more visual words, and that speakers use these words more frequently. In comparison, English features fewer distinct taste and smell words, and speakers tend to verbalize taste, and particularly smell, concepts less frequently. The fact that precisely those sensory modalities that are more frequently talked about also have more semantic distinctions supports the view that English perceptual vocabulary is adapted towards the communicative needs of its speakers.

\section{Methods}

\subsection{Using modality norms to characterize sensory modalities}

We utilized native speaker ratings to quantify the degree to which a word was visual, auditory, tactile, gustatory or olfactory. Such "modality norms" have been collected by many researchers (including Lynott \& Connell, 2009, 2013; Speed \& Majid, 2017; van Dantzig, Cowell, Zeelenberg, \& Pecher, 2011; Winter, 2016a). The basic task was innovated by Lynott and Connell (2009), who asked 55 native speakers of British English to rate a set of 423 property words (adjectives) on a scale from 0 to 5 on each of the five sensory modalities. The word yellow, for example, received an average rating of 4.9 on its "visual strength", compared to ratings of $0,0.2,0.1$ and 0.1 for tactile, auditory, gustatory and olfactory strength respectively. The norms can be considered "well-calibrated" with respect to studying the intersection of language and perception because they have been shown to correspond meaningfully to a number of behavioral measures (Connell \& Lynott, 2010, 2012, 2014, 2016; Speed \& Majid, 2017; van Dantzig et al., 2011) and linguistic patterns (Louwerse \& Connell, 2011; Winter, 2016a, 2016b; Winter, Perlman, Perry, \& Lupyan, 2017).

Here, we use the adjective norms collected by Lynott and Connell (2009) $(N=423)$, the noun norms by Lynott and Connell (2013) $(N=400)$ and the verb norms by Winter (2016a) $(N=300)$. Our total data set comprises 1123 words. For ease of discussion, we focus our analyses of token frequencies on the SUBTLEX corpus of movie subtitles (see Brysbaert \& New, 2009 for arguments in favor of using this corpus). However, we replicate our analyses with several old and new frequency lists that are commonly used in psycholinguistics and linguistics (several are taken from the English Lexicon Project, Balota et al., 2007). These corpus-based word frequency lists include Kučera and Francis (Kučera \& Francis, 1967), the Hyperspace Analogue of Language (HAL, Lund \& Burgess, 1996), SUBTLEX-UK (Keuleers, Lacey, Rastle, \& Brysbaert, 2012), CELEX (Baayen, Piepenbrock, \& van Rijn, 1993) and the British National Corpus (Leech, 1992). These different corpora contain texts from multiple time spans and feature different linguistic registers and different dialects (both British English and American 
English). For the register analysis, we additionally use the Corpus of Contemporary American English (COCA, Davies, 2008), which has 5 distinct linguistic registers: fiction, academic language, newspapers, magazines and spoken language. For the historical analysis, we used the Corpus of Historical American English (COHA).

\subsection{Statistical analyses}

All statistical analyses were performed within the R programming environment version 3.1.1 ( $R$ Core Team, 2016) and several $R$ packages. ${ }^{1}$ In line with recommendations for reproducible research (Gentleman \& Lang, 2007; Mesirov, 2010; Munafò et al., 2017; Peng, 2011), all data and analysis code is made publicly available and can be retrieved on GitHub: http://github.com/bodowinter/visual_ dominance/.

We performed two sets of analyses. In the first set of analyses, we used each word's "dominant modality" (see Lynott \& Connell, 2009), based on each word's maximum perceptual strength rating. For example, the word yellow was classified as visual because its highest perceptual strength rating was for the visual modality, and the words rough and fragrant were classified as tactile and olfactory, respectively. In our categorical analyses, we focused on highly exclusive words, that is, words that were closely tied to a given modality. For this, we used Lynott and Connell's (2009) measure of "modality exclusivity" (defined as the range of perceptual strength ratings divided by the sum of perceptual strength ratings), which measures the degree to which a word is relatively more multisensory (e.g., harsh is $11 \%$ exclusive) or unisensory (e.g., purple is $90 \%$ exclusive). In the second set of analyses, we used the continuous perceptual strength ratings. Here, words were allowed to be multisensory and their association with particular sensory modalities was a matter of degree rather than kind, e.g., the word yellow is relatively more visual than rough, which itself is relatively more visual than fragrant.

\section{Results}

\subsection{Type frequencies}

We first examined the visual dominance hypothesis by examining how many words are associated with vision, in contrast to the other sensory modalities. This has previously been examined in part by Lynott and Connell (2009, 2013) and Winter (2016b), but an integrated analysis is presented here to allow comparison with token frequencies. To compute discrete counts, we used the dominant modality classification. Fig. 1 shows the proportion of word types for each part-of-speech. For the property concepts from Lynott and Connell (2009), there are 205 visual adjectives (48\%), 70 touch adjectives (17\%), 68 sound adjectives (16\%), 54 taste adjectives (13\%) and 26 smell adjectives (6\%). A chisquare test across these counts reveals they reliably deviate from a uniform distribution, $\chi^{2}(4)=228.78, p<0.0001$. The nouns from Lynott and Connell (2013) are also non-uniformly distributed, $\chi^{2}(4)=$ 1036.2, $p<0.0001$; with 336 sight-related nouns (84\%), 42 soundrelated nouns $(10 \%), 14$ touch-related nouns (4\%), 6 taste-related nouns (2\%) and 0 smell-related nouns ( $0 \%)$. Finally, the same applies to the verbs from Winter (2016a), $\chi^{2}(4)=133.07, p<0.0001$ : there are 102 sight-related verbs (84\%), 71 sound-related verbs (10\%), 101

\footnotetext{
${ }^{1}$ The R packages dplyr version 0.7.1 (Wickham, Francois, Henry, \& Müller, 2017), readr 1.0.0 (Wickham, Hester, \& Francois, 2016), tidyr 0.6.0 (Wickham, 2016b), magrittr 1.5 (Bache \& Wickham, 2014), stringr version 1.1.0 (Wickham, 2016a) and png 0.1-7 (Urbanek, 2013) were used for data carpentry and visualization. The package MASS 7.3.45 (Venables \& Ripley, 2002) was used for fitting negative binomial regression models, and the R package pscl 1.49 (Jackman, 2015) was used to assess overdispersion to motivate negative binomial models. Finally, the package car 2.1.3. (Fox \& Weisberg, 2011) was used to assess collinearity. The packages mgcv 1.8.15 (Wood, 2006) and itsadug 2.2 (van Rij, Wieling, Baayen, \& van Rijn, 2016) were used for computing and visualizing generalized additive models.
}
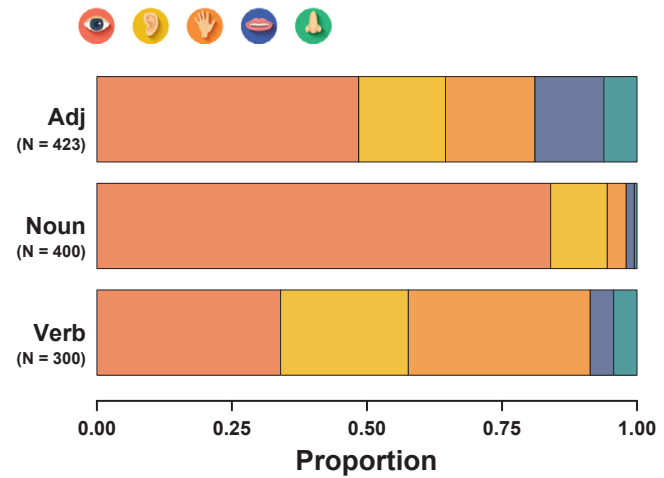

Fig. 1. Stacked bar plot of type frequency proportions for each sensory modality per part-of-speech.

touch-related verbs (4\%), 13 taste-related verbs (2\%) and 13 smell-related verbs (0\%). Analysis of standardized adjusted Pearson residuals (see Levshina, 2015: 220-221) reveals that vision is reliably over-represented for adjectives $(+14.64)$, nouns $(+32.00)$ and verbs $(+6.06)$. Taste is consistently under-represented for adjectives $(-3.72)$, nouns $(-9.25)$ and verbs $(-6.78)$. The same applies to smell, which is consistently under-represented for adjectives $(-7.12)$, nouns $(-9.75)$ and verbs $(-6.78)$. For touch and sound there are mixed results. For adjectives, there are relatively fewer auditory $(-2.02)$ and tactile words $(-1.77)$ than is expected by chance; the same applies to nouns (sound: -4.75 ; touch: -8.25$)$. For verbs, there are relatively more auditory $(+1.59)$ and tactile $(+5.92)$ words.

The analyses so far looked at the entire set of words, including highly multisensory words. To assess whether the multisensory nature of certain perceptual words impacts our results, we additionally assessed type frequencies for only those words that were above the 80th percentile of the modality exclusivity measure. This analysis confirms the established pattern, with $59 \%$ of all adjectives being visual for this reduced dataset of highly unisensory words (next: $31 \%$ sound). For nouns, $80 \%$ of the highly unisensory words were visual (next: $19 \%$ sound). Only for verbs did this data exclusion measure change the ranking with respect to vision: There were $40 \%$ sound concepts, $17 \%$ touch and $15 \%$ visual concepts for highly unisensory concepts. The fact that sound concepts are overall more exclusive (Lynott \& Connell, 2009, 2013) may bias the results towards the auditory modality. Still, by and large, the data suggest that vision is the most lexically differentiated, even if only the most unisensory words per modality are analyzed. All in all, these results show that the English language is most lexically differentiated in the visual modality, and our results furthermore show a distinct lack of gustatory and olfactory vocabulary for adjectives, nouns, and verbs.

\subsection{Token frequencies}

Next, we examined whether each visual word is also used more frequently. An initial analysis revealed that there was an overall negative relationship between modality exclusivity and token frequency taken from SUBTLEX US (log estimate: $-0.7, S E=0.31, p=0.03$ ). Since the five sensory modalities systematically differ with respect to their modality exclusivity (Lynott \& Connell, 2009, 2013), this could bias the analysis against more exclusive modalities. Moreover, when considering whether certain types of sensory experience are more likely to be verbalized, it is best to use words that are highly indicative of that sensory modality, i.e., words that have high modality exclusivity. Therefore, in the following analysis, we focused on the 10 most exclusive words per sensory modality per part-of-speech category. This resulted in a data set of 50 verbs, 50 adjectives, and 38 nouns (the unequal number comes from the fact that there are fewer than 10 nouns for taste and smell in the Lynott \& Connell, 2013 dataset). 
Descriptive averages for these highly exclusive words revealed that dominantly visual words were used on average 12,841 times each in SUBTLEX, followed by auditory words (5503 times), touch words (4653), taste words (1504), and smell words (939). The rank ordering for vision was the same regardless of whether one splits up these average counts for adjectives, verbs or nouns. So words that are (exclusively) highly visual were also used more frequently for all the partof-speech categories considered in this study.

To model token counts inferentially, we regressed the raw counts from the SUBTLEX corpus onto the five different perceptual strength ratings (each rating scale was z-scored). We added parts-of-speech as a categorical control variable. Token counts were modeled using negative binomial regression, which is a form of the generalized linear model used for count data, similar to Poisson/loglinear models (for more details about this form of regression, see Zuur, Ieno, Walker, Saveliev, \& Smith, 2009, and O'hara \& Kotze, 2010). Just like Poisson models, the dependent measure for this regression technique is counts (in this case, raw word frequencies), and the average rate of an event (in this case, the rate of a word occurring) is tied to the predictor variables via the log link function. In contrast to Poisson regression, negative binomial models include one additional parameter that estimates dispersion, which is desirable in the case of excess variance ("overdispersion"), a common characteristic of complex and highly variable linguistic data.

All five continuous perceptual strength measures were entered into the same model. ${ }^{2}$ The negative binomial regression model revealed a reliable positive effect on word token frequency only for visual strength ratings ( $\log$ estimate: $+0.66, S E=0.18, p=0.0002$ ), and tactile strength ratings $(+0.44, S E=0.17, p=0.009)$. There were no reliable effects for auditory strength ratings $(+0.34, S E=0.19, p=0.076$ ), gustatory strength ratings $(-0.33, S E=0.19, p=0.076)$, or olfactory strength ratings $(-0.37, S E=0.21, p=0.08)$. Fig. 2 shows the negative binomial regression coefficients for each sensory modality. All in all, we find words are more frequent if they correspond more strongly to visual content and tactile content, a tendency which is absent for sound, taste, and smell.

To assess the generality of our results-i.e., to test whether the same pattern holds across words-we performed leave-one-out influence diagnostics for the two significant results reported above (i.e., visual and tactile strength ratings). For this, we refit the negative binomial regression model without the first word in the dataset, the second word etc. For visual strength ratings, the $z$-scored beta coefficients ranged from +0.56 to +0.76 . There was no word exclusion that resulted in a non-significant result. For touch, the coefficients ranged from +0.30 to +0.56 . If the word feel was excluded, the correlation between haptic strength ratings and frequency ceded to be statistically reliable $(p=0.07)$.

Finally, we assessed whether there was a distinct contribution of part-of-speech on the main result for vision. We fit another negative binomial regression model for just visual strength ratings, with an interaction term for parts-of-speech. This analysis revealed a reliable main effect of visual strength ratings, $\chi^{2}(1)=31.31, p<0.0001$, as well as a reliable interaction between visual strength ratings and partsof-speech, $\chi^{2}(2)=9.55, p=0.008$. The frequency slope was strongest for adjectives $(+1.43, S E=0.26)$, with the noun $(-1.17, S E=0.44)$ and verb $(-0.83, S E=0.34)$ interaction terms adjusting this slope downwards, although not reversing, the main correlation between frequency and visual strength ratings.

Overall, then, these analyses show that visual words really are more frequent across the board than words from the other sensory modalities.

\footnotetext{
${ }^{2}$ Because certain perceptual strength ratings are correlated with each other (such as taste and smell ratings, see Louwerse \& Connell, 2011; Lynott \& Connell, 2009), we assessed collinearity via variance inflation factors (see Zuur, Ieno, \& Elphick, 2010). These were found to be low, indicating no problem with collinearity. Overdispersion tests indicated a significant degree of overdispersion, which justifies our choice of negative binomial regression models over Poisson models.
}

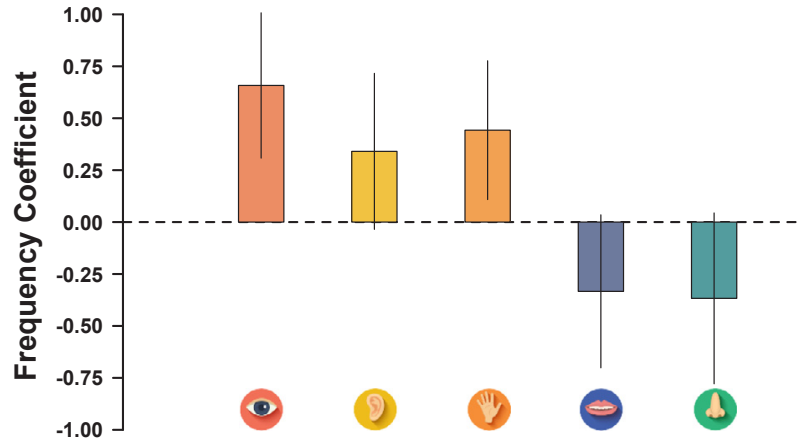

Fig. 2. Frequency coefficients from negative binomial model for associations between continuous perceptual strength ratings and SUBTLEX word tokens; error bars show 95\% confidence intervals.

\subsection{Stability of token frequencies}

Next, we examined whether the patterns observed so far are a register-stable and time-stable property of the English language. Do our previous results generalize across different contexts of use? For ease of visualization, we use cumulative frequencies, which combine type counts and token counts, using the full datasets (across all parts of speech). This also gives a rough estimate of how much each sensory modality is talked about in relation to the others. Fig. 3 shows cumulative frequencies across seven different corpora of American English (SUBTLEX, COCA, HAL, Kučera-Francis) and British English (SUBTLEXUK, CELEX, BNC). As can be seen, vision is consistently more frequent, regardless of the particular corpus or variety of English.

Separate negative binomial regression models were fitted to the frequencies from each corpus, with all five perceptual strength ratings treated as continuous predictors (z-scored). (Here again, we only use the 10 most exclusive words per sensory modality per part-of-speech.) There was a reliable positive association between frequency and visual strength ratings for all corpora (all p's of visual strength coefficients $<0.001$ ). The only other sensory modality for which perceptual strength ratings were reliably associated with frequency was the tactile modality (all p's of tactile strength coefficients $<0.05$ ); however, the association was consistently weaker than for vision. For vision, the largest z-scored coefficient was obtained for COCA $(+1.09, S E=0.23$, $p<0.0001)$ and the smallest for Kučera-Francis $(+0.59, S E=0.13$, $p<0.0001)$. For the same two corpora, the tactile modality had coefficients of only $+0.77(S E=0.22, p=0.0004)$ and +0.27 ( $S E=0.13, p=0.035$ ), respectively. For the other sensory modalities, the only reliable effects were the following: in three out of the seven corpora, there was a statistically reliable positive association between auditory strength and word frequency (COCA: +0.50, $S E=0.25$; HAL: $+0.37, S E=0.17$; SUBTLEX UK: $+0.40, S E=0.19$, all $p$ 's $<0.05$ ). In three out of the seven corpora, there was also a statistically reliable negative association between olfactory strength and word frequency (COCA: $-0.92, S E=0.27$; HAL: -0.59 ; $S E=0.18$; Kučera-Francis: $-0.35, S E=0.17)$. Gustatory strength did not correlate with frequency in any of the corpora (all p's $>0.05$ ).

Next, we assessed the generality of our results with respect to linguistic registers. We expected that certain linguistic registers differentially relate to particular sensory modalities. For example, taste and smell words have been shown to be used more often in emotional textual contexts (Winter, 2016a). Given this, it is possible that visual dominance might not hold across the board. Fig. 4 shows, however, that the pattern of visual dominance in cumulative frequencies is consistent across the five linguistic registers represented in the Corpus of Contemporary American English: academic language, fiction, magazines, news, and spoken language.

Separate negative binomial regression models were fitted to the 


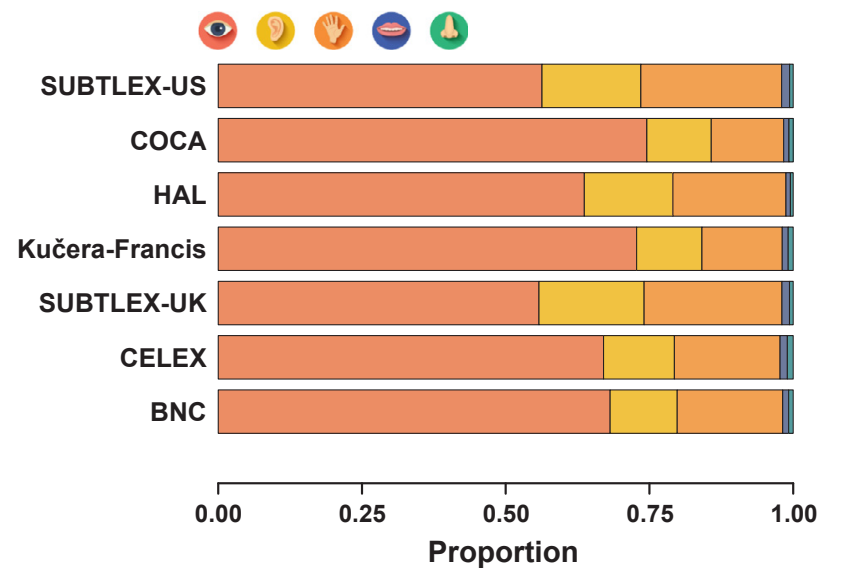

Fig. 3. Cumulative frequency proportions per modality for seven different corpora.

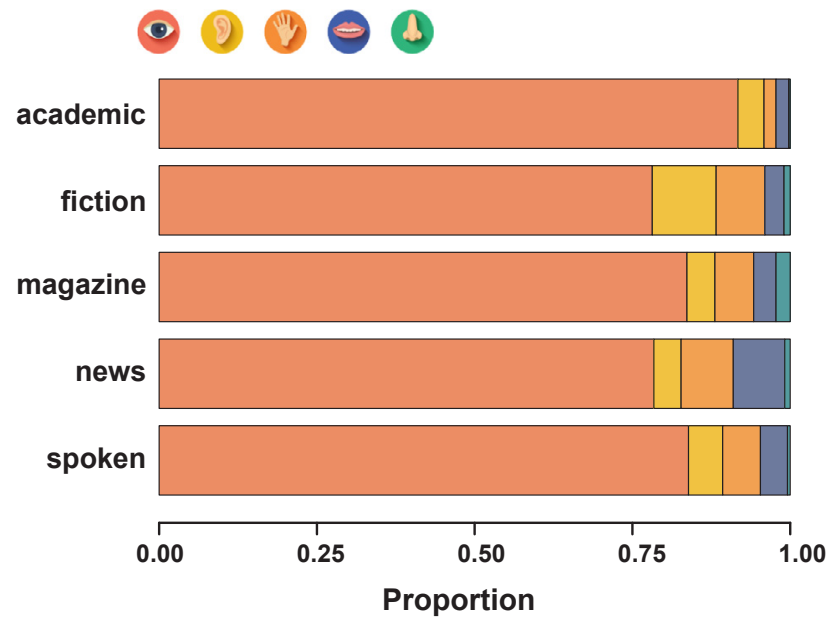

Fig. 4. Cumulative frequency proportions per modality for five different registers from the Corpus of Contemporary American English (Davies, 2008).

frequencies from each linguistic register, with all five perceptual strength ratings treated as continuous predictors (z-scored). These models are not designed to determine whether there are differences between linguistic registers, but instead whether the effect of visual strength ratings is consistent for all registers. For academic language, there were reliable positive effects for visual $(+0.50, S E=0.07$, $p<0.0001)$ and auditory strength ratings $(+0.54, S E=0.07$, $p<0.0001)$, as well as reliable negative effects for gustatory $(-0.22$, $S E=0.1, p=0.02)$ and olfactory strength ratings $(-0.26, S E=0.1$, $p=0.007)$. For fiction, there were reliable positive effects for visual $(+0.48, S E=0.06, p<0.0001)$ and tactile strength ratings $(+0.19$, $S E=0.06, p=0.001$ ), as well as reliable negative effects for gustatory strength ratings $(-0.17, S E=0.08, p=0.044)$. For magazines, there were reliable positive effects for visual $(+0.49, S E=0.06$, $p<0.0001)$, tactile $(+0.15, S E=0.06, p=0.01)$ and auditory strength ratings $(+0.14, S E=0.06, p=0.02)$. For newspapers, there were reliable positive effects for visual $(+0.5, S E=0.06, p<0.0001)$ and auditory strength ratings $(+0.27, S E=0.06, p<0.0001)$, as well as a reliable negative effect for olfactory strength ratings $(-0.22$, $S E=0.09, p=0.01$ ). Finally, for spoken language there were also reliable positive effects for visual $(+0.34, S E=0.07, p<0.001)$ and auditory strength ratings $(+0.45, S E=0.07, p<0.001)$, as well as a reliable negative effect for olfactory strength ratings $(-0.4, S E=0.1$, $p=0.0001$ ). So each of the five registers showed a reliable positive association between word frequencies and visual strength ratings. In addition, token frequencies displayed a reliable positive association for

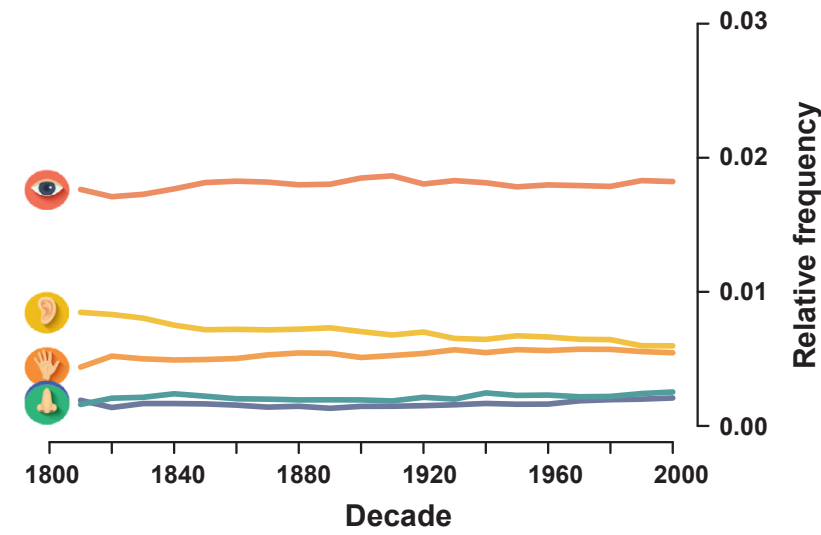

Fig. 5. Descriptive average frequencies (relativized to total word count for the corresponding decade) for each modality, based on the 10 most exclusive words per modality per parts-of-speech; data from the Corpus of Historical American English.

four out of the five registers for auditory strength ratings, and two registers for tactile strength ratings. On the other hand, token frequencies displayed a reliable negative association in two registers for gustatory strength ratings, and three for olfactory strength ratings. In sum, across different registers we find the same evidence of visual dominance.

Finally, we focused on the temporal dimension: is visual dominance a recent phenomenon or is it a time-stable property of the English language? Fig. 5 shows the frequency of sensory adjectives over 200 years of American English, with data taken from the Corpus of Historical American English (COHA). The plot shows descriptive means of relative frequencies. We computed relative frequencies because the total word count differs per decade. We computed the linear slope for each word and subjected these slopes as a dependent measure to a simple linear regression analysis with the single categorical predictor "modality". Neither the total set of words, $F(4,1118)=1.50, p=0.2$, nor the 10 most exclusive words per modality, $F(4,133)=0.59$, $p=0.67$ showed a statistically reliable modality effect on the timedependent frequency slopes. So, there is no substantial evidence for an overall increase or decrease in the relative frequency with which certain modalities are used across the last 200 years of American English ${ }^{3}$. This suggests a substantive degree of diachronic stability of the synchronic patterns we reported above.

\section{Discussion}

A number of studies demonstrate how the lexicon of English and other languages are optimized for communication about various conceptual domains, including color, ice and snow, and kinship (e.g., Gibson et al., 2017; Kemp \& Regier, 2012; Regier et al., 2016). At the same time, a number of researchers have suggested that the English

\footnotetext{
${ }^{3}$ We also fit mixed generalized additive models (GAMs), a method suitable for time series analysis, especially in the presence of nonlinear patterns (Wood, 2006; see Winter \& Wieling, 2016 \& Sóskuthy, 2017 for tutorial introductions; see Smith \& Levy, 2013 and Sóskuthy \& Hay, 2017 for cognitive science applications). The GAMs included a predictor variable for whether the word was visual or not, as well as by-word time-dependent factor smooths (random effects). We fit models separately for each parts-of-speech. For the 10 most exclusive words, there was a reliable parametric effect of vision versus not vision for adjectives $(+0.007, S E=0.002, t=3.9, p=0.0001)$, and verbs $(+0.02, S E=0.01$, $t=2.33, p=0.02)$, but not nouns $(+0.008, S E=0.005, t=1.7, p=0.09)$. When a fivelevel modality factor was fitted, the visual slope was statistically reliable only for adjectives $(+0.006, S E=0.002, t=2.68, p=0.007)$, but not for nouns $(+0.005$, $S E=0.006, t=0.83, p=0.41)$ or verbs $(+0.02, S E=0.01, t=1.55, p=0.12)$. In addition, there were reliable time-vision interactions for adjectives (edf $=3.5, F=7.52$, $p<0.001)$, verbs ( $e d f=1.0, F=13.87, p=0.0002)$ and nouns $(e d f=2.75, F=8.54$, $p<0.001$ ), with the relative frequency of visual adjectives and verbs slightly increasing over time, and the relative frequency of visual nouns slightly decreasing over time.
} 
language, and other languages (e.g., Viberg, 1983; San Roque et al., 2015), exhibit visual dominance in usage and vocabulary structure. Here, we showed that the perceptual vocabulary of English strongly supports the visual dominance hypothesis-across parts-of-speech, registers, varieties of English, and across time. Vocabulary for concepts related to vision features more unique word types, and importantly, these words are also used more frequently. This suggests that precisely those perceptual domains that English speakers frequently talk about have greater expressive potential in terms of the structure of the vocabulary. ${ }^{4}$

The observed correspondence between type and token frequency is far from trivial. As an alternative baseline, if all other factors were equal, we would expect sensory domains with greater lexical differentiation to be less frequent because there are more visual words to choose from, thus lowering the average frequency of each word across this larger set of unique word types. That is, because English speakers have more visual words to choose from, the frequency of each individual word would be expected to be lower. Given this tradeoff, it is particularly noteworthy that despite a higher type frequency, we also found a higher token frequency for visual concepts.

Our general findings were supported by a series of statistical analyses of 1123 English words, including 423 adjectives, 400 nouns, and 300 verbs. Within this dataset, we found strong and consistent evidence of visual dominance, as well as for the diminished vocabulary and frequency for the sensory modalities of taste and smell. The long-held idea in linguistics that vision is the most differentiated sensory modality (see Levinson \& Majid, 2014) was thus confirmed in a quantitative fashion, and in a way that generalizes beyond previous studies which have been limited to perception verbs and a few isolated word types (Viberg, 1983; Viberg, 1993; San Roque et al., 2015; but see Strik Lievers \& Winter, 2018).

The fact that visual dominance in the lexicon-as well as the diminution of taste and smell-holds across nouns, verbs, and adjectives is particularly important. Although these grammatical categories are best defined distributionally (see Baker \& Croft, 2017), each of these parts-of-speech is associated with particular semantic prototypes: timevarying events, processes and actions for verbs; time-stable objects and entities for nouns; and properties of intermediate temporal stability for adjectives (Givón, 1979, 2001 [1984]; Gärdenfors, 2014; Langacker, 2008; Murphy, 2010; Strik Lievers \& Winter, 2018). Thus, nouns, verbs and adjectives serve quite different functions within a language, both morphosyntactically (their role within sentences), as well as semantically (the typical types of meaning they denote). Given this variability, it is particularly striking that vision is dominant for all lexical categories. Although Strik Lievers and Winter (2018) discuss some interesting relative differences in how the different sensory domains are expressed in particular parts-of-speech categories (in particular, auditory concepts are relatively more differentiated in the verbal domain), the results discussed here show a marked degree of visual dominance across the major parts-of-speech. This suggests that vision is more important regardless of whether one looks at property descriptions (adjectives), reference to objects (nouns) or descriptions of perceptual activities (verbs). The pattern of visual dominance in word frequencies was, however, strongest when looking at adjectives, arguably the class of words that is most directly about the content of sensory perception (e.g., blue, bright, murky, mottled).

Of course, it has to be noted that the register differences investigated in this study correspond to fairly coarse-grained differences

\footnotetext{
${ }^{4}$ The fact that the perceptual vocabulary is "optimized" (geared towards) communicative efficiency does not imply that it is in any ways "optimal", something which is difficult to measure and establish anyway (see Kinsella \& Marcus, 2009; Marcus \& Davis, 2013 , 2015). Instead, we view the patterns obtained here as broadly in line with usagebased theories of language which state that the structures of languages, including the structures of their lexicons, are shaped by communicative use (e.g., Barlow \& Kemmer, 2000; Bybee \& Hopper, 2001).
}

in usage. The results we obtained here characterize large chunks of the English language, but they are compatible with other sensory modalities being relatively more important in more specialized discourse, such as cook books and movie reviews. Strik Lievers (2015) discusses Patrick Süsskind's novel The Perfume (Ger. 'Das Parfum') (see also Popova, 2003), and how in this particular novel, smell is the most frequent target domain of perceptual metaphors. Even if the visual dominance is reversed in particular specialist texts, the fact that we find visual dominance in large register-balanced corpora suggests that across-the-board, word frequencies exhibit visual dominance.

The flipside of the visual dominance we established with our English data is that some sensory modalities have diminished expressive capabilities and reduced frequencies. Our results fit particularly well with what has been said about smell, which has been characterized as a "muted sense" (Olofsson \& Gottfried, 2015; Yeshurun \& Sobel, 2010) due to its lack of lexical differentiation (see also Buck, 1949: chap. 15; Levinson \& Majid, 2014), at least in many Western languages (cf. de Valk, Wnuk, Huisman, \& Majid, 2017; Majid, 2015; Majid \& Burenhult, 2014; Majid \& Kruspe, 2018; O’Meara \& Majid, 2016; Wnuk \& Majid, 2014). We found fewer words were rated high on olfactory strength, and olfactory strength was negatively associated with word token frequencies, i.e., the more a word related to smell, the less frequent it was. Moreover, we found relatively few smell words to begin with. With respect to both type and token frequencies, taste behaved similarly to smell. This shows that although taste is important to speakers within certain domains (e.g., Korsmeyer, 1999)—as is smell-it fades in importance compared to vision when taking into consideration language use in a broader context. The finding that taste and smell were both similarly infrequent fits the notion that these perceptual modalities are highly similar (Auvray \& Spence, 2008; Rozin, 1982; Spence, Smith, \& Auvray, 2015; Stevenson \& Oaten, 2010), and that their vocabularies are generally associated in language (Classen, 1993, chap. 3; Louwerse \& Connell, 2011; Winter, 2016a, 2016b).

A potential methodological concern arises from how the words were selected for the modality norming study. Clearly, the way a word list is chosen could impact the results. However, there are several reasons why sampling considerations cannot "explain away" the finding of visual dominance in English. First, the three word lists featured in this study were sampled in different ways: whereas Lynott and Connell (2009) was hand-compiled, Lynott and Connell (2013) was randomly assembled, and Winter's (2016a) list contained a random ( $\sim 40 \%)$ and a hand-compiled part ( $\sim 60 \%)$. In addition, Strik Lievers (2015) hand compiled a list of words, and the results replicate there too (see Github repository). The fact that the same pattern of visual dominance was found across these different lists, shows this result is robust to type of sampling.

A pressing question is to what extent do the findings observed here generalize across cultures? It has been reported that certain cultures value smell relatively more, and correspondingly have more elaborated smell vocabularies (e.g., Burenhult \& Majid, 2011; Majid \& Burenhult, 2014; Majid \& Kruspe, 2018; Wnuk \& Majid, 2014). Majid et al. (in press) find that in terms of codability (i.e., consensus in how to describe sensory experiences) there is no universal hierarchy of the senses across a diverse set of unrelated languages. If a universal hierarchy is forced, then taste and color emerge as the most codable domains across languages, followed by shape, sound and touch, and finally, smell. This suggests that cultural factors can override the patterns we find for English here. However, it is also possible that the fit between type and token frequencies reported here is something that carries over to languages which have different rankings of the senses. This is something that requires systematic investigation.

To conclude, we found that asymmetries between the senses as revealed in perception, culture, and neuroanatomy correspond to asymmetries between the senses in the perceptual vocabulary of English, as well as to how this perceptual vocabulary is deployed in natural language use. In terms of both type and token frequencies, the senses are 
not created equal: there is demonstrable visual dominance in the usage of sensory words, as well as in the composition of the sensory lexicon. The finding that type and token frequencies are correlated supports the view that the perceptual vocabulary of English is oriented towards the needs of its speakers, with precisely those sensory domains more frequently verbalized also being the most lexically differentiated, allowing for more nuanced distinctions and more expressive descriptions of perceptual content. Our finding thus provides a prime example of how the composition of vocabularies of languages reflects communicative need.

\section{Acknowledgments}

We thank Jennifer Arnold and three reviewers (including Diane Pecher and Kyle Mahowald) for helpful comments. We thank Florent Perek for his help with the COHA data. This work was funded in part by the Netherlands Organization for Scientific Research: NWO VICI grant "Human olfaction at the intersection of language, culture and biology".

\section{References}

Alais, D., \& Burr, D. (2004). The ventriloquist effect results from near-optimal bimodal integration. Current Biology, 14, 257-262.

Auvray, M., \& Spence, C. (2008). The multisensory perception of flavor. Consciousness and Cognition, 17, 1016-1031.

Baayen, R. H., Piepenbrock, R., \& van Rijn, H. (1993). The CELEX lexical data base on \{CD-ROM\}.

Baker, M., \& Croft, W. (2017). Lexical categories: Legacy, lacuna, and opportunity for functionalists and formalists. Annual Review of Linguistics, 3, 179-197.

Balota, D. A., Yap, M. J., Hutchison, K. A., Cortese, M. J., Kessler, B., Loftis, B., Treiman, R. (2007). The English Lexicon Project. Behavior Research Methods, 39 $445-459$.

Barlow, M., \& Kemmer, S. (Eds.). (2000). Usage-Based Models of Language. Stanford: CSLI Publications.

Berlin, B., \& Kay, P. (1969). Basic color terms: Their universality and evolution. Berkeley: University of California Press.

Brower, D. (1947). The experimental study of imagery: II. The relative predominance of various imagery modalities. The Journal of General Psychology, 37(2), 199-200.

Brysbaert, M., \& New, B. (2009). Moving beyond Kučera and Francis: a critical evaluation of current word frequency norms and the introduction of a new and improved word frequency measure for American English. Behavior Research Methods, 41, 977-990.

Buck, C. D. (1949). A dictionary of selected synonyms in the principal Indo-European languages: A contribution to the history of ideas. Chicago: University of Chicago Press.

Burenhult, N., \& Majid, A. (2011). Olfaction in Aslian ideology and language. The Senses and Society, 6, 19-29.

Bybee, J., \& Hopper, P. (Eds.). (2001). Frequency and the emergence of linguistic structure. Amsterdam: John Benjamins.

Caplan, D. (1973). A note on the abstract readings of verbs of perception. Cognition, 2, 269-277.

Classen, C. (1993). Worlds of sense: Exploring the senses in history and across cultures London: Routledge.

Classen, C. (1997). Foundations for an anthropology of the senses. International Social Science Journal, 49, 401-412.

Connell, L., \& Lynott, D. (2010). Look but don't touch: Tactile disadvantage in processing modality-specific words. Cognition, 115(1), 1-9.

Connell, L., \& Lynott, D. (2012). Strength of perceptual experience predicts word processing performance better than concreteness or imageability. Cognition, 125, $452-465$.

Connell, L., \& Lynott, D. (2014). I see/hear what you mean: Semantic activation in visual word recognition depends on perceptual attention. Journal of Experimental Psychology: General, 143(2), 527-533.

Connell, L., \& Lynott, D. (2016). Do we know what we're simulating? Information loss on transferring unconscious perceptual simulation to conscious imagery. Journal of Experimental Psychology: Learning Memory, and Cognition, 42(8), 1218-1232.

Cook, R. S., Kay, P., \& Regier, T. (2005). The world color survey database. In H. Cohen, \& C. Lefebvre (Eds.). Handbook of categorization in cognitive science (pp. 223-241). Amsterdam: Elsevier.

Davies, M. (2008). The Corpus of Contemporary American English: 450 million words, 1990-present. Available online at < http://corpus.byu.edu/coca/ > .

de Valk, J. M.de, Wnuk, E., Huisman, J. L. A., \& Majid, A. (2017). Odor-color associations differ with verbal descriptors for odors: a comparison of three linguistically diverse groups. Psychonomic Bulletin \& Review, 24, 1171-1179.

Drury, H. A., Van Essen, D. C., Anderson, C. H., Lee, C. W., Coogan, T. A., \& Lewis, J. W. (1996). Computerized mappings of the cerebral cortex: a multiresolution flattening method and a surface-based coordinate system. Journal of Cognitive Neuroscience, 8 , $1-28$.

Evans, N., \& Wilkins, D. (2000). In the mind's ear: The semantic extensions of perception verbs in Australian languages. Language, 76, 546-592.

Fox, J., \& Weisberg, S. (2011). An R companion to applied regression. Thousand Oaks CA:
Sage.

Gärdenfors, P. (2014). The geometry of meaning: Semantics based on conceptual spaces Cambridge, MA: MIT Press.

Gentleman, R., \& Lang, D. (2007). Statistical analyses and reproducible research. Journal of Computational and Graphical Statistics, 16, 1-23.

Gibson, E., Futrell, R., Jara-Ettinger, J., Mahowald, K., Bergen, L., Ratnasingam, S., ... Conway, B. R. (2017). Color naming across languages reflects color use. Proceedings of the National Academy of Sciences, 114, 10785-10790.

Givón, T. (1979). On understanding grammar. Orlando, FL: Academic Press.

Givón, T. (2001 [1984]). Syntax: A functional-typological introduction, Vol. 1. Amsterdam-Philadelphia: Benjamins.

Griffin, L. D. (2006). The basic colour categories are optimal for classification. Journal of the Royal Society: Interface, 3, 71-85.

Hay, J. C., \& Pick, H. L. (1966). Visual and proprioceptive adaptation to optical displacement of the visual stimulus. Journal of Experimental Psychology, 71, 150-158.

Hidaka, S., \& Shimoda, K. (2014). Investigation of the effects of color on judgments of sweetness using a taste adaptation method. Multisensory Research, 27, 189-205.

Ibarretxe-Antuñano, I. (2008). Vision metaphors for the intellect: Are they really crosslinguistic? Atlantis, 15-33.

Jackman, S. (2015). pscl: Classes and methods for R developed in the political science computational laboratory, Stanford University. R package version 1.4.9.

Keller, A. (2016). Philosophy of olfactory perception. Cham: Palgrave Macmillan.

Kemp, C., \& Regier, T. (2012). Kinship categories across languages reflect general communicative principles. Science, 336(6084), 1049-1054.

Keuleers, E., Lacey, P., Rastle, K., \& Brysbaert, M. (2012). The British Lexicon Project: Lexical decision data for 28,730 monosyllabic and disyllabic English words. Behavior Research Methods, 44, 287-304.

Kinsella, A. R., \& Marcus, G. F. (2009). Evolution, perfection, and theories of language. Biolinguistics, 3, 186-212.

Korsmeyer, C. (1999). Making sense of taste: Food and philosophy. Ithaca: Cornell University Press.

Kosslyn, S. M., Seger, C., Pani, J. R., \& Hillger, L. A. (1990). When is imagery used in everyday life? A diary study. Journal of Mental Imagery, 14(3-4), 131-152.

Kučera, H., \& Francis, W. (1967). Computational analysis of present day American English. Providence, RI: Brown University Press.

Langacker, R. W. (2008). Cognitive grammar: A basic introduction. Oxford, UK: Oxford University Press.

Leech, G. (1992). 100 million words of English: the British National Corpus (BNC). Language Research, 28, 1-13.

Levinson, S. C., \& Majid, A. (2014). Differential ineffability and the senses. Mind \& Language, 29, 407-427.

Levshina, N. (2015). How to do linguistics with R: Data exploration and statistical analysis. Amsterdam: John Benjamins.

Louwerse, M., \& Connell, L. (2011). A taste of words: Linguistic context and perceptual simulation predict the modality of words. Cognitive Science, 35, 381-398.

Lund, K., \& Burgess, C. (1996). Producing high-dimensional semantic spaces from lexical co-occurrence. Behavior Research Methods, Instruments, \& Computers, 28, 203-208.

Lynott, D., \& Connell, L. (2009). Modality exclusivity norms for 423 object properties. Behavior Research Methods, 41, 558-564.

Lynott, D., \& Connell, L. (2013). Modality exclusivity norms for 400 nouns: The relationship between perceptual experience and surface word form. Behavior Research Methods, 45, 516-526.

Majid, A. (2015). Cultural factors shape olfactory language. Trends in Cognitive Sciences, $19,629-630$.

Majid, A., \& Burenhult, N. (2014). Odors are expressible in language, as long as you speak the right language. Cognition, 130, 266-270.

Majid, A., Roberts, S. G., Cilissen, L., Emmorey, K., Nicodemus, B., O'Grady, L., ... Levinson, S. C. (in press). The differential coding of perception in the world's languages. Proceedings of the National Academy of Sciences.

Majid, A., \& Kruspe, N. (2018). Hunter-gatherer olfaction is special. Current Biology, 28, 409-413.

Marcus, G. F., \& Davis, E. (2015). Still searching for principles: A response to Goodman et al. (2015). Psychological Science, 26, 542-544.

Marcus, G. F., \& Davis, E. (2013). How robust are probabilistic models of higher-level cognition? Psychological Science, 24, 2351-2360.

Matlock, T. (1989). Metaphor and the grammaticalization of evidentials. Proceedings of the 15th annual meeting of the berkeley linguistics society (pp. 215-225). Berkeley: Berkeley Linguistics Society.

Mesirov, J. P. (2010). Computer science. Accessible reproducible research. Science, 327, 5964.

Morrot, G., Brochet, F., \& Dubourdieu, D. (2001). The color of odors. Brain and Language, 79, 309-320.

Munafò, M. R., Nosek, B. A., Bishop, D. V., Button, K. S., Chambers, C. D., du Sert, N. P., et al. (2017). A manifesto for reproducible science. Nature Human Behaviour, 1, 0021.

Murphy, M. L. (2010). Lexical meaning. Cambridge: Cambridge University Press.

O'hara, R. B., \& Kotze, D. J. (2010). Do not log-transform count data. Methods in Ecology and Evolution, 1, 118-122.

O'Meara, C., \& Majid, A. (2016). How changing lifestyles impact Seri smellscapes and smell language. Anthropological Linguistics, 58, 107-131.

Olofsson, J. K., \& Gottfried, J. A. (2015). The muted sense: neurocognitive limitations of olfactory language. Trends in Cognitive Sciences, 19, 314-321.

Palmer, S. E. (1999). Vision science: Photons to phenomenology. Cambridge, MA: MIT Press.

Peng, R. D. (2011). Reproducible research in computational science. Science, 334 1226-1227.

Pick, H. L., Warren, D. H., \& Hay, J. C. (1969). Sensory conflict in judgments of spatial direction. Perception \& Psychophysics, 6, 203-205. 
Popova, Y. (2003). 'The fool sees with his nose': Metaphoric mappings in the sense of smell in Patrick Süskind's Perfume. Language and Literature, 12, 135-151.

R Core Team (2016). R: A language and environment for statistical computing. R Foundation for Statistical Computing, Vienna, Austria.

Regier, T., Carstensen, A., \& Kemp, C. (2016). Languages support efficient communication about the environment: words for snow revisited. PLOS ONE, 11, e0151138.

Rock, I., \& Victor, J. (1964). Vision and touch: an experimentally created conflict between the two senses. Science, 143, 594-596.

Rozin, P. (1982). "Taste-smell confusions" and the duality of the olfactory sense. Attention, Perception, \& Psychophysics, 31, 397-401.

San Roque, L., Kendrick, K. H., Norcliffe, E., Brown, P., Defina, R., Dingemanse, M., ... Majid, A. (2015). Vision verbs dominate in conversation across cultures, but the ranking of non-visual verbs varies. Cognitive Linguistics, 26, 31-60.

Shermer, D. Z., \& Levitan, C. A. (2014). Red hot: The crossmodal effect of color intensity on perceived piquancy. Multisensory Research, 27, 207-223.

Smith, N. J., \& Levy, R. (2013). The effect of word predictability on reading time is logarithmic. Cognition, 128, 302-319.

Sóskuthy, M. (2017). Generalised Additive Mixed Models for dynamic analysis in linguistics: A practical introduction. arXiv: 1703.05339.

Sóskuthy, M., \& Hay, J. (2017). Changing word usage predicts changing word durations in New Zealand English. Cognition, 166, 298-313.

Speed, L. J., \& Majid, A. (2017). Dutch modality exclusivity norms: Simulating perceptual modality in space. Behavior Research Methods, 1-15 [e-publication ahead of print].

Spence, C., Parise, C., \& Chen, Y. C. (2012). The Colavita Visual Dominance Effect. In W. M. Murray (Ed.). The neural bases of multisensory processes (pp. 529-556). Boca Raton, FL: CRC Press.

Spence, C., Smith, B., \& Auvray, M. (2015). Confusing tastes and flavours. In D. Stokes, M. Matthen, \& S. Biggs (Eds.). Perception and its modalities (pp. 247-274). Oxford: Oxford University Press.

Stevenson, R. J., \& Oaten, M. J. (2010). Sweet odours and sweet tastes are conflated in memory. Acta Psychologica, 134, 105-109.

Stokes, D., \& Biggs, S. (2015). The dominance of the visual. In D. Stokes, M. Matthen, \& S. Biggs (Eds.). Perception and its modalities (pp. 350-378). Oxford: Oxford University Press.

Strik Lievers, F. (2015). Synaesthesia: A corpus-based study of cross-modal directionality. In R. Caballero, \& C. Paradis (Eds.). Functions of language, sensory perceptions in language and cognition (pp. 69-95). Amsterdam: John Benjamins.

Strik Lievers, F., \& Winter, B. (2018). Sensory language across lexical categories. Lingua, 204, 45-61.

Sweetser, E. (1990). From etymology to pragmatics: Metaphorical and cultural aspects of semantic structure. Cambridge: Cambridge University Press.

Urbanek, S. (2013). png: Read and write PNG images. R package version 0.1-7 < https:// CRAN.R-project.org $/$ package $=$ png $>$.

van Dantzig, S., Cowell, R. A., Zeelenberg, R., \& Pecher, D. (2011). A sharp image or a sharp knife: Norms for the modality-exclusivity of 774 concept-property items.
Behavior Research Methods, 43, 145-154.

van Rij, J., Wieling, M., Baayen, R., \& van Rijn, H. (2016). itsadug: Interpreting time series and autocorrelated data using GAMMs. R package version 2.2 .

Venables, W. N. \& Ripley, B. D. (2002). Modern Applied Statistics with S. New York: Springer.

Viberg, A. (1983). The verbs of perception: A typological study. Linguistics, 21, 123-162.

Viberg, Å. (1993). Crosslinguistic perspectives on lexical organization and lexical progression. In K. Hyltenstam,\& A. Viberg (Eds.), Progression and Regression in Language: Sociocultural, Neuropsychological and Linguistic Perspectives (pp. 340-385). Cambridge: Cambridge University Press.

Warriner, A. B., \& Kuperman, V. (2015). Affective biases in English are bi-dimensional. Cognition and Emotion, 29, 1147-1167.

Welch, R. B., \& Warren, D. H. (1980). Immediate perceptual response to intersensory discrepancy. Psychological Bulletin, 88, 638-667.

Wickham, H. (2016a). Stringr: Simple, Consistent Wrappers for Common String Operations. R package version 1.1.0. https://CRAN.R-project.org/package = stringr.

Wickham, H. (2016b). tidyr: Easily Tidy Data with 'spread()' and 'gather()' Functions. R package version 0.6.0. https://CRAN.R-project.org/package = tidyr.

Wickham, H., Hester, J., \& Francois, R. (2016). readr: Read Tabular Data. R package version 1.0.0. https://CRAN.R-project.org/package $=$ readr.

Wickham, H., Francois, R., Henry, L., \& Müller, K. (2017). dplyr: A Grammar of Data Manipulation. R package version 0.7.1. < https://CRAN.R-project.org/package = dplyr $>$.

Winter, B. (2016a). Taste and smell words form an affectively loaded part of the English lexicon. Language, Cognition and Neuroscience, 31, 975-988.

Winter, B. (2016b). The Sensory Structure of the English LexiconPhD Thesis. Merced: University of California.

Winter, B., Perlman, M., Perry, L. K., \& Lupyan, G. (2017). Which words are most iconic? Iconicity in English sensory words. Interaction Studies, 18, 433-454.

Winter, B., \& Wieling, M. (2016). How to analyze linguistic change using mixed models, Growth Curve Analysis and Generalized Additive Modeling. Journal of Language Evolution, 1, 7-18.

Wnuk, E., \& Majid, A. (2014). Revisiting the limits of language: The odor lexicon of Maniq. Cognition, 131, 125-138.

Wood, S. N. (2006). Generalized additive models: An introduction with R. New York: Chapman and Hall/CRC.

Yendrikhovskij, S. (2001). Computing color categories from statistics of natural images. Journal of Imaging Science and Technology, 45, 409-417.

Yeshurun, Y., \& Sobel, N. (2010). An odor is not worth a thousand words: from multidimensional odors to unidimensional odor objects. Annual Review of Psychology, 61, 219-241.

Zuur, A. F., Ieno, E. N., \& Elphick, C. S. (2010). A protocol for data exploration to avoid common statistical problems. Methods in Ecology and Evolution, 1, 3-14.

Zuur, A. F., Ieno, E. N., Walker, N. J., Saveliev, A. A., \& Smith, G. M. (2009). Mixed Effects Models and Extensions in Ecology with R. New York: Springer. 